Research Article

\title{
A study on antibiotic sensitivity pattern of bacterial isolates in the intensive care unit of a tertiary care hospital in Eastern India
}

\author{
Chaitali Pattanayak $^{\mathrm{a} *}$, Sunil K. Patanaik ${ }^{\mathrm{b}}$, Pratyay Pratim Datta ${ }^{\mathrm{a}}$, Parbaty Panda
}

${ }^{\text {a } D e p a r t m e n t ~ o f ~ P h a r m a c o l o g y, ~ H i-~}$ Tech Medical College and Hospital, Bhubaneswar, Odisha, India,

${ }^{\mathrm{b}}$ Consultant Surgeon, Sun

Hospital, Cuttack, Odisha, India

Received: 1 January 2013

Accepted: 27 January 2013

*Correspondence to:
Dr. Chaitali Pattanayak,
Email:
cpattanayak2010@ rediffmail.com
(C) 2013 Pattanayak C et al. This is
an open-access article distributed
under the terms of the Creative
Commons Attribution License,
which permits unrestricted use,
distribution, and reproduction in
any medium, provided the original
work is properly cited.

*Correspondence to:

Dr. Chaitali Pattanayak,

Email:

(C) 2013 Pattanayak C et al. This is an open-access article distributed under the terms of the Creative which permits unrestricted use, distribution, and reproduction in work is properly cited

\begin{abstract}
Background: Nosocomial infections are one of the leading causes of morbidity and mortality in hospitalized patients especially the critically ill patients in the intensive care unit (ICU) where a large number of drugs are administered to the patient' which in turn leads to the generation of antibiotic resistant pathogens. The present study was conducted to identify the prevalence of predominant bacterial microorganisms and their drug sensitivity and resistance in ICU of a teaching hospital in Eastern India.

Methods: A retrospective record based study was conducted in the ICU of HiTech Medical College and Hospital, Odisha, Eastern India from November, 2011 to October, 2012. Patients who were clinically suspected of having acquired any infection after 48 hours of admission to the ICUs were included in the study. The clinically suspected laboratory samples were collected from the patients and subjected to testing and antibiotic sensitivity.

Results: The rate of nosocomial infection was $28.2 \%$. Urinary tract infection was the most common infection $(54.9 \%)$. The predominant isolate was $E$. coli (52.7\%) followed by $P$. mirabilis (15.4\%) and Ps aeruginosa (13.2\%). E. coli was highly sensitive to Polymyxin B, Gatifloxacin and Ceftriaxone and showed high degree of resistance to Cephalexin, Cefadroxil, Tobramycin and Prulifloxacin.

Conclusions: Most of the bacterial isolates were resistant to third generation Cephalosporins and Aminoglycosides. Regular surveillance of antibiotic susceptibility pattern, judicious use of antibiotics are very important for reducing the nosocomial infection rate and antimicrobial resistance.
\end{abstract}

Keywords: Nosocomial infection, Intensive care unit, Antibiotic resistance, Bacterial isolates, Antibiotic susceptibility

\section{INTRODUCTION}

Throughout the world multi-drug resistant nosocomial infections are one of the leading causes of death and morbidity amongst hospitalized patients, accounting a major burden on patients and public health system of any country. ${ }^{1,2}$

Intensive care unit (ICU) is one of the potential sources of nosocomial infections even in countries where extensive infection control measures are routinely implemented. The international study of infections in ICU, which was conducted in 2007, demonstrated that the patients who had longer ICU stays had higher rates of infection, especially infections due to resistant
Staphylococci, Acinetobacter, Pseudomonas species, Candida species. $^{3}$

The rate of nosocomial infections in the ICU is rising, mainly because of increasing use of invasive procedures which are performed in the ICU. The therapeutic interventions which are associated with infectious complications include indwelling catheters, sophisticated life support, intravenous fluid therapy, prosthetic devices, immunosuppressive therapy, and use of broad spectrum antibiotics leading to a spectrum of multi-drug resistant pathogens, which contributed to the evolution of the problem of nosocomial infections. ${ }^{4}$ Moreover, the ICU mortality of infectious patients is more than twice that of non-infected patients. ${ }^{5}$ 
Antibiotic resistance, a global concern, is particularly pressing in developing nations, including India. ${ }^{6}$ Antibiotic overuse and misuse partly due to incorrect diagnosis, irrational and counterfeit antibiotic market combinations, and irregular consumption due to either wrong prescription or poor compliance all contribute to the widespread drug resistance among the hospital acquired organisms. ${ }^{7}$ The patterns of organisms causing infections and their antibiotic resistance pattern vary widely from one country to another, as well as from one hospital to other. Presently, India lacks any local or national level surveillance program, to guide the stakeholders on actual prevalence of resistance. ${ }^{8}$

The aim of the present study was to identify the prevalence of predominantly isolated bacterial microorganisms and their drug resistance patterns for the patients admitted in the ICU in a private multispecialty hospital in Bhubaneswar, Eastern India.

\section{METHODS}

Study setting: A retrospective record based study was carried out based on reports of bacteria isolates from the ICU of a private multispecialty hospital in Eastern India with 15 beds for medical ICU.

Study period: Samples of the patients admitted in the ICU during November, 2011 to October 2012 were included in the study.

Study sample: The Centre for Disease Control and Prevention (CDC) defines ICU associated infections as those that occur after $48 \mathrm{hrs}$ of ICU admission or within $48 \mathrm{hrs}$ after transfer from an ICU. ${ }^{9}$

In the present study, patients admitted in ICU during the study period, who were clinically suspected of having acquired any infection after $48 \mathrm{hrs}$ of admission to ICU transfer to the ICU were not included. The following signs and symptoms were considered:

- $\quad$ Fever $\geq 38^{\circ} \mathrm{C}$, leucocytes $\geq 10,000 /$ cu mm.

- New infiltrates on chest X-ray, persistent tracheal aspirates or secretions.

- Turbid urine, suprapubic tenderness, dysuria and burning micturition, thrombophlebitis.

Depending on the clinical suspicions, laboratory samples like urine, sputum, pus, swab, blood, body fluids, Foley's catheter tips, ET tips, CVP line tips were collected from the patients.

Study tool: Identification of all causative microorganisms was performed by standard microbiologic methods. Susceptibility testing was performed using disk diffusion method.
Other information regarding the patient including age, gender, date of admission was also collected from the case records of the patients.

Statistical analysis: After collection of data it was double entered in Microsoft Excel sheet and verified. A clean datasheet was generated and copied into SPSS sheet (SPSS version 16.0). After this the whole analysis was done in SPSS (version 16.0).

\section{RESULTS}

During the 12 month study period, a total of 347 patients were admitted to the ICU, of which 98(28.2\%) had clinically suspected nosocomial infections. A total of 312 patients' samples were analyzed, out of which $182(58.3 \%)$ samples were positive for growth of organisms. The growth positive samples included CVP line tips $2(1.1 \%)$, ET tube 3(1.64\%), urine 93(51.1\%), blood 13(7.1\%), Foley's catheter tips 13(7.1\%), body fluids $2(1.1 \%)$, pus $10(5.5 \%)$, sputum10 $(5.5 \%)$ and swabs $42(23.1 \%)$ as shown in Table 1 .

Escherichia coli (E. coli) 96(52.7\%) was the most frequently isolated bacteria, followed by Proteus mirabilis (P. mirabilis) 28(15.4\%), Pseudomonas aeruginosa (Ps. aeruginosa) 24 (13.2\%), Candida albicans 12 (6.6\%), Staphylococcus aureus (S. aureus) 10 $(5.5 \%)$, Klebsiella pneumoniae (K. pneumoniae) 6 (3.3\%), Enterococcus fecalis (E. fecalis) 4 (2.2\%).

Though, on gram stain Candida was also identified, the bacterial samples were subjected to testing and antibiotic sensitivity.

Antibiotic sensitivity pattern of major six bacterial isolates is as per Table No.2. E. coli was most commonly sensitive to Polymyxin B (100\%), Gatifloxacin (56.7\%) and Ceftriaxone $(51.6 \%), P$. mirabilis was sensitive to Gatifloxacin (47.4\%), Ps. aeruginosa was sensitive to Gatifloxacin (80\%) and Netilmicin $(50 \%)$, S. aureus was sensitive to Vancomycin (100\%) and Linezolid (100\%), K. pneumoniae was sensitive to Sparfloxacin (100\%), Levofloxacin (100\%), Piperacillin-Tazobactum (100\%) and E. fecalis to Amoxicillin-Clavulanic acid (100\%).

The isolated bacteria showed a very high rate of resistance to the Cephalosporins namely Cefuroxime, Ceftazidime, Cefixime, Cefpodoxime.

\section{DISCUSSION}

Health care acquired infections have been associated with substantial morbidity, mortality and increased health care costs. An integrated infection control program can reduce the incidence of infection by as much as $30 \%$ and reduce the health care costs. ${ }^{10}$

The present study included the types and antibiotic susceptibility pattern of bacterial organisms isolated from 
Table 1: Frequency of microorganisms isolated various specimens.

\begin{tabular}{|c|c|c|c|c|c|c|c|c|c|c|c|c|c|}
\hline \multirow[b]{2}{*}{ Organism } & \multicolumn{13}{|c|}{ Specimen } \\
\hline & Urine & Blood & Pus & Sputum & $\begin{array}{l}\text { Throat } \\
\text { swab }\end{array}$ & $\begin{array}{l}\text { Trachea } \\
\text { l swab }\end{array}$ & $\begin{array}{l}\text { Pleural } \\
\text { fluid }\end{array}$ & $\begin{array}{l}\text { Wound } \\
\text { swab }\end{array}$ & $\begin{array}{l}\text { CVP } \\
\text { line tip }\end{array}$ & $\begin{array}{l}\text { Catheter } \\
\text { tip }\end{array}$ & $\begin{array}{l}\text { ET } \\
\text { tube }\end{array}$ & $\begin{array}{l}\text { Drain } \\
\text { fluid }\end{array}$ & Total \\
\hline Candida & $\begin{array}{l}4 \\
(4.3)\end{array}$ & $\begin{array}{l}0 \\
(0)\end{array}$ & $\begin{array}{l}0 \\
(0)\end{array}$ & $\begin{array}{l}2 \\
(20)\end{array}$ & $\begin{array}{l}0 \\
(0)\end{array}$ & $\begin{array}{l}0 \\
(0)\end{array}$ & $\begin{array}{l}0 \\
(0)\end{array}$ & $\begin{array}{l}6 \\
(18.2)\end{array}$ & $\begin{array}{l}0 \\
(0)\end{array}$ & $\begin{array}{l}0 \\
(0)\end{array}$ & $\begin{array}{l}0 \\
(0)\end{array}$ & $\begin{array}{l}0 \\
(0)\end{array}$ & $\begin{array}{l}12 \\
(6.6)\end{array}$ \\
\hline Commensal & $\begin{array}{l}0 \\
(0)\end{array}$ & $\begin{array}{l}0 \\
(0)\end{array}$ & $\begin{array}{l}0 \\
(0)\end{array}$ & $\begin{array}{l}0 \\
(0)\end{array}$ & $\begin{array}{l}2 \\
(100)\end{array}$ & $\begin{array}{l}0 \\
(0)\end{array}$ & $\begin{array}{l}0 \\
(0)\end{array}$ & $\begin{array}{l}0 \\
(0)\end{array}$ & $\begin{array}{l}0 \\
(0)\end{array}$ & $\begin{array}{l}0 \\
(0)\end{array}$ & $\begin{array}{l}0 \\
(0)\end{array}$ & $\begin{array}{l}0 \\
(0)\end{array}$ & $\begin{array}{l}2 \\
(1.1)\end{array}$ \\
\hline E. coli & $60(64.5)$ & $\begin{array}{l}9 \\
(69.2)\end{array}$ & $\begin{array}{l}4 \\
(40)\end{array}$ & $\begin{array}{l}4 \\
(40)\end{array}$ & $\begin{array}{l}0 \\
(0)\end{array}$ & $\begin{array}{l}3 \\
(42.9)\end{array}$ & $\begin{array}{l}1 \\
(100)\end{array}$ & $\begin{array}{l}10 \\
(30.3)\end{array}$ & $\begin{array}{l}2 \\
(100)\end{array}$ & $\begin{array}{l}2 \\
(28.6)\end{array}$ & $\begin{array}{l}0 \\
(0)\end{array}$ & $\begin{array}{l}1 \\
(100)\end{array}$ & $\begin{array}{l}96 \\
(52.7)\end{array}$ \\
\hline Klebsiella spp. & $\begin{array}{l}0 \\
(0)\end{array}$ & $\begin{array}{l}0 \\
(0)\end{array}$ & $\begin{array}{l}0 \\
(0)\end{array}$ & $\begin{array}{l}4 \\
(40)\end{array}$ & $\begin{array}{l}0 \\
(0)\end{array}$ & $\begin{array}{l}2 \\
(28.6)\end{array}$ & $\begin{array}{l}0 \\
(0)\end{array}$ & $\begin{array}{l}0 \\
(0)\end{array}$ & $\begin{array}{l}0 \\
(0)\end{array}$ & $\begin{array}{l}0 \\
(0)\end{array}$ & $\begin{array}{l}0 \\
(0)\end{array}$ & $\begin{array}{l}0 \\
(0)\end{array}$ & $\begin{array}{l}6 \\
(3.3)\end{array}$ \\
\hline Proteus spp. & $\begin{array}{l}9 \\
(9.7)\end{array}$ & $\begin{array}{l}0 \\
(0)\end{array}$ & $\begin{array}{l}4 \\
(40)\end{array}$ & $\begin{array}{l}0 \\
(0)\end{array}$ & $\begin{array}{l}0 \\
(0)\end{array}$ & $\begin{array}{l}2 \\
(28.6)\end{array}$ & $\begin{array}{l}0 \\
(0)\end{array}$ & $\begin{array}{l}7 \\
(21.2)\end{array}$ & $\begin{array}{l}0 \\
(0)\end{array}$ & $\begin{array}{l}3 \\
(42.9)\end{array}$ & $\begin{array}{l}3 \\
(100)\end{array}$ & $\begin{array}{l}0 \\
(0)\end{array}$ & $\begin{array}{l}28 \\
(15.4)\end{array}$ \\
\hline $\begin{array}{l}\text { Pseudomonas } \\
\text { spp. }\end{array}$ & $\begin{array}{l}14 \\
(15.1)\end{array}$ & $\begin{array}{l}0 \\
(0)\end{array}$ & $\begin{array}{l}0 \\
(0) \\
\end{array}$ & $\begin{array}{l}0 \\
(0)\end{array}$ & $\begin{array}{l}0 \\
(0)\end{array}$ & $\begin{array}{l}0 \\
(0)\end{array}$ & $\begin{array}{l}0 \\
(0)\end{array}$ & $\begin{array}{l}8 \\
(24.2)\end{array}$ & $\begin{array}{l}0 \\
(0)\end{array}$ & $\begin{array}{l}2 \\
(28.6)\end{array}$ & $\begin{array}{l}0 \\
(0)\end{array}$ & $\begin{array}{l}0 \\
(0)\end{array}$ & $\begin{array}{l}24 \\
(13.2)\end{array}$ \\
\hline Staph. spp. & $\begin{array}{l}2 \\
(2.2) \\
\end{array}$ & $\begin{array}{l}4 \\
(30.8)\end{array}$ & $\begin{array}{l}2 \\
(20) \\
\end{array}$ & $\begin{array}{l}0 \\
(0) \\
\end{array}$ & $\begin{array}{l}0 \\
(0) \\
\end{array}$ & $\begin{array}{l}0 \\
(0) \\
\end{array}$ & $\begin{array}{l}0 \\
(0) \\
\end{array}$ & $\begin{array}{l}2 \\
(6.1) \\
\end{array}$ & $\begin{array}{l}0 \\
(0)\end{array}$ & $\begin{array}{l}0 \\
(0) \\
\end{array}$ & $\begin{array}{l}0 \\
(0) \\
\end{array}$ & $\begin{array}{l}0 \\
(0) \\
\end{array}$ & $\begin{array}{l}10 \\
(5.5)\end{array}$ \\
\hline $\begin{array}{l}\text { Enterococcus } \\
\text { spp. }\end{array}$ & $\begin{array}{l}4 \\
(4.3)\end{array}$ & $\begin{array}{l}0 \\
(0)\end{array}$ & $\begin{array}{l}0 \\
(0)\end{array}$ & $\begin{array}{l}0 \\
(0)\end{array}$ & $\begin{array}{l}0 \\
(0)\end{array}$ & $\begin{array}{l}0 \\
(0)\end{array}$ & $\begin{array}{l}0 \\
(0)\end{array}$ & $\begin{array}{l}0 \\
(0)\end{array}$ & $\begin{array}{l}0 \\
(0)\end{array}$ & $\begin{array}{l}0 \\
(0)\end{array}$ & $\begin{array}{l}0 \\
(0)\end{array}$ & $\begin{array}{l}0 \\
(0)\end{array}$ & $\begin{array}{l}4 \\
(2.2)\end{array}$ \\
\hline Total & $\begin{array}{l}93 \\
(100)\end{array}$ & $\begin{array}{l}13 \\
(100)\end{array}$ & $\begin{array}{l}10 \\
(100)\end{array}$ & $\begin{array}{l}10 \\
(100)\end{array}$ & $\begin{array}{l}2 \\
(100)\end{array}$ & $\begin{array}{l}7 \\
(100)\end{array}$ & $\begin{array}{l}1 \\
(100)\end{array}$ & $\begin{array}{l}33 \\
(100)\end{array}$ & $\begin{array}{l}2 \\
(100)\end{array}$ & $\begin{array}{l}7 \\
(100)\end{array}$ & $\begin{array}{l}3 \\
(100)\end{array}$ & $\begin{array}{l}1 \\
(100)\end{array}$ & $\begin{array}{l}182 \\
(100)\end{array}$ \\
\hline
\end{tabular}


Table 2: Antibiotic sensitivity pattern of predominant micro-organisms isolated from patients.

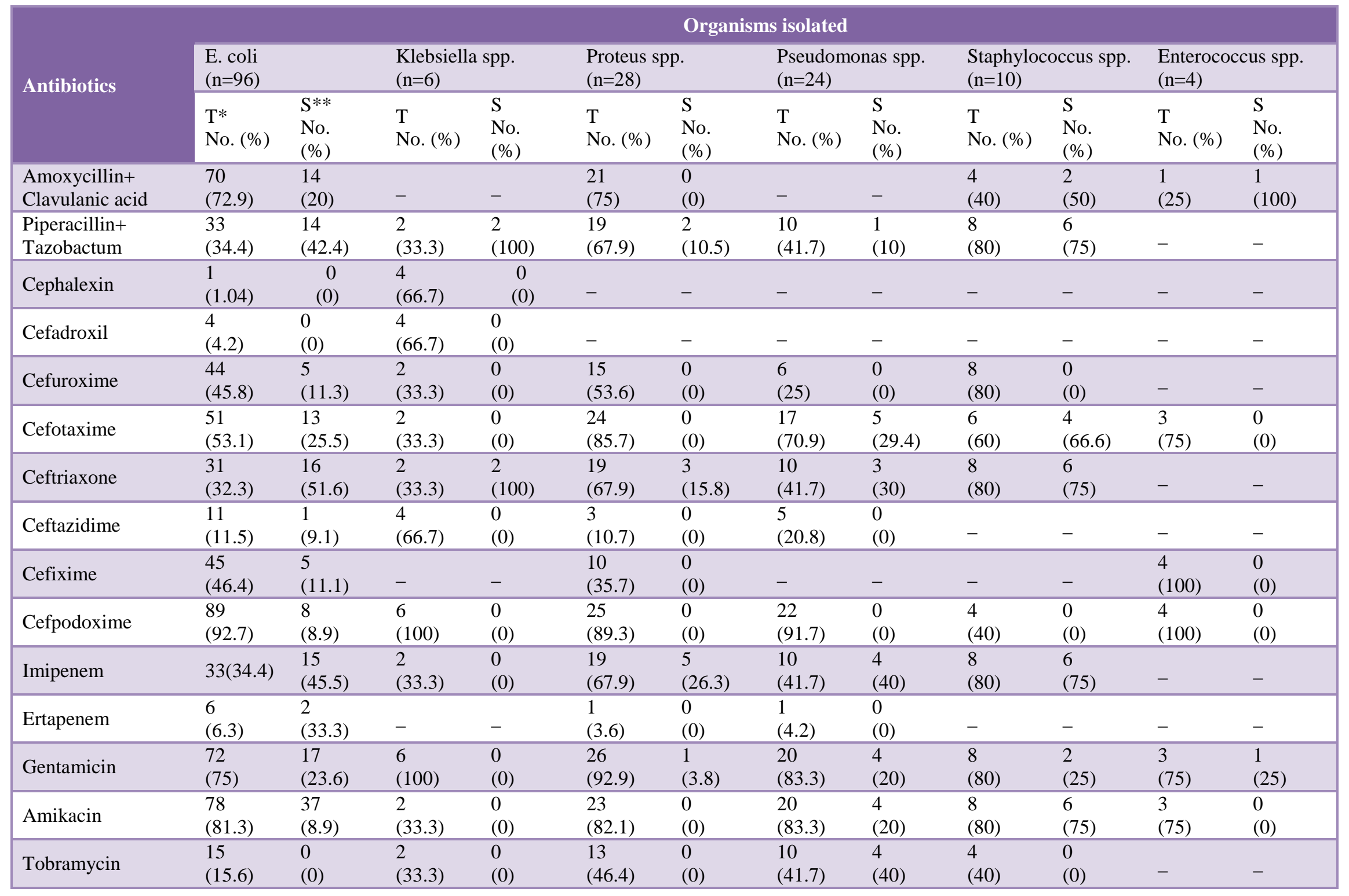


Pattanayak C et al. Int J Basic Clin Pharmacol. 2013 Apr;2(2):153-159

\begin{tabular}{|c|c|c|c|c|c|c|c|c|c|c|c|c|}
\hline Netilmicin & $\begin{array}{l}17 \\
(17.7)\end{array}$ & $\begin{array}{l}5 \\
(29.4)\end{array}$ & $\begin{array}{l}4 \\
(66.7)\end{array}$ & $\begin{array}{l}0 \\
(0)\end{array}$ & $\begin{array}{l}4 \\
(14.3)\end{array}$ & $\begin{array}{l}0 \\
(0)\end{array}$ & $\begin{array}{l}4 \\
(16.7)\end{array}$ & $\begin{array}{l}2 \\
(50)\end{array}$ & $\begin{array}{l}2 \\
(20)\end{array}$ & $\begin{array}{l}2 \\
(100)\end{array}$ & $\begin{array}{l}1 \\
(25)\end{array}$ & $\begin{array}{l}0 \\
(0)\end{array}$ \\
\hline Ciprofloxacin & $\begin{array}{l}68 \\
(70.8)\end{array}$ & $\begin{array}{l}10 \\
(14.7)\end{array}$ & $\begin{array}{l}4 \\
(66.7)\end{array}$ & $\begin{array}{l}4 \\
(100)\end{array}$ & $\begin{array}{l}9 \\
(32.1)\end{array}$ & $\begin{array}{l}2 \\
(22.2)\end{array}$ & $\begin{array}{l}14 \\
(58.3)\end{array}$ & $\begin{array}{l}2 \\
(14.3)\end{array}$ & $\begin{array}{l}2 \\
(20)\end{array}$ & $\begin{array}{l}2 \\
(100)\end{array}$ & $\begin{array}{l}4 \\
(100)\end{array}$ & $\begin{array}{l}0 \\
(0)\end{array}$ \\
\hline Norfloxacin & $\begin{array}{l}3 \\
(3.1)\end{array}$ & $\begin{array}{l}0 \\
(0)\end{array}$ & - & - & - & - & - & - & - & - & - & - \\
\hline Ofloxacin & $\begin{array}{l}87 \\
(90.6)\end{array}$ & $\begin{array}{l}17 \\
(19.5)\end{array}$ & $\begin{array}{l}2 \\
(33.3)\end{array}$ & $\begin{array}{l}0 \\
(0)\end{array}$ & $\begin{array}{l}28 \\
(100)\end{array}$ & $\begin{array}{l}3 \\
(10.7)\end{array}$ & $\begin{array}{l}24 \\
(100)\end{array}$ & $\begin{array}{l}4 \\
(16.7)\end{array}$ & $\begin{array}{l}10 \\
(100)\end{array}$ & $\begin{array}{l}6 \\
(60)\end{array}$ & $\begin{array}{l}4 \\
(100)\end{array}$ & $\begin{array}{l}3 \\
(75)\end{array}$ \\
\hline Levofloxacin & $\begin{array}{l}93 \\
(96.9)\end{array}$ & $\begin{array}{l}32 \\
(34.4)\end{array}$ & $\begin{array}{l}4 \\
(66.7)\end{array}$ & $\begin{array}{l}4 \\
(100)\end{array}$ & $\begin{array}{l}25 \\
(89.3)\end{array}$ & $\begin{array}{l}6 \\
(24)\end{array}$ & $\begin{array}{l}23 \\
(95.8)\end{array}$ & $\begin{array}{l}8 \\
(34.8)\end{array}$ & $\begin{array}{l}8 \\
(80)\end{array}$ & $\begin{array}{l}6 \\
(75)\end{array}$ & $\begin{array}{l}4 \\
(100)\end{array}$ & $\begin{array}{l}0 \\
(0)\end{array}$ \\
\hline Prulifloxacin & $\begin{array}{l}53 \\
(55.2)\end{array}$ & $\begin{array}{l}0 \\
(0)\end{array}$ & - & - & $\begin{array}{l}9 \\
(32.1)\end{array}$ & $\begin{array}{l}0 \\
(0)\end{array}$ & $\begin{array}{l}11 \\
(45.9)\end{array}$ & $\begin{array}{l}0 \\
(0)\end{array}$ & $\begin{array}{l}2 \\
(20)\end{array}$ & $\begin{array}{l}0 \\
(0)\end{array}$ & $\begin{array}{l}4 \\
(100)\end{array}$ & $\begin{array}{l}0 \\
(0)\end{array}$ \\
\hline Sparfloxacin & $\begin{array}{l}6 \\
(6.3)\end{array}$ & $\begin{array}{l}3 \\
(50)\end{array}$ & $\begin{array}{l}4 \\
(66.7)\end{array}$ & $\begin{array}{l}4 \\
(100)\end{array}$ & $\begin{array}{l}1 \\
(3.6)\end{array}$ & $\begin{array}{l}0 \\
(0)\end{array}$ & - & - & - & - & - & - \\
\hline Gatifloxacin & $\begin{array}{l}30 \\
(31.3)\end{array}$ & $\begin{array}{l}17 \\
(56.7)\end{array}$ & $\begin{array}{l}2 \\
(33.2)\end{array}$ & $\begin{array}{l}2 \\
(100)\end{array}$ & $\begin{array}{l}19 \\
(67.9)\end{array}$ & $\begin{array}{l}9 \\
(47.4)\end{array}$ & $\begin{array}{l}10 \\
(41.7)\end{array}$ & $\begin{array}{l}8 \\
(80)\end{array}$ & $\begin{array}{l}8 \\
(80)\end{array}$ & $\begin{array}{l}6 \\
(75)\end{array}$ & - & - \\
\hline Gemifloxacin & $\begin{array}{l}49 \\
(51)\end{array}$ & $\begin{array}{l}7 \\
(14.3) \\
\end{array}$ & - & - & $\begin{array}{l}9 \\
(32.1)\end{array}$ & $\begin{array}{l}2 \\
(22.2)\end{array}$ & $\begin{array}{l}14 \\
(58.3)\end{array}$ & $\begin{array}{l}0 \\
(0)\end{array}$ & $\begin{array}{l}2 \\
(20)\end{array}$ & $\begin{array}{l}2 \\
(100)\end{array}$ & $\begin{array}{l}4 \\
(100)\end{array}$ & $\begin{array}{l}0 \\
(0)\end{array}$ \\
\hline Nitrofurantoin & $\begin{array}{l}58 \\
(60.4)\end{array}$ & $\begin{array}{l}29 \\
(50)\end{array}$ & - & - & - & - & - & - & - & - & - & - \\
\hline Azithromycin & $\begin{array}{l}90 \\
(93.8)\end{array}$ & $\begin{array}{l}40 \\
(44.4)\end{array}$ & $\begin{array}{l}6 \\
(100)\end{array}$ & $\begin{array}{l}4 \\
(66.7)\end{array}$ & $\begin{array}{l}26 \\
(92.9)\end{array}$ & $\begin{array}{l}2 \\
(7.7)\end{array}$ & $\begin{array}{l}22 \\
(91.7)\end{array}$ & $\begin{array}{l}10 \\
(45.5)\end{array}$ & $\begin{array}{l}10 \\
(100)\end{array}$ & $\begin{array}{l}6 \\
(60)\end{array}$ & $\begin{array}{l}4 \\
(100)\end{array}$ & $\begin{array}{l}0 \\
(0)\end{array}$ \\
\hline Lincomycin & - & - & - & - & - & - & - & - & $\begin{array}{l}2 \\
(20)\end{array}$ & $\begin{array}{l}2 \\
(100)\end{array}$ & - & - \\
\hline Vancomycin & - & - & - & - & - & - & - & - & $\begin{array}{l}8 \\
(80)\end{array}$ & $\begin{array}{l}8 \\
(100)\end{array}$ & $\begin{array}{l}4 \\
(100)\end{array}$ & $\begin{array}{l}1 \\
(25)\end{array}$ \\
\hline Teicoplanin & - & - & - & - & - & - & - & - & $\begin{array}{l}6 \\
(60)\end{array}$ & $\begin{array}{l}2 \\
(33.3)\end{array}$ & - & - \\
\hline Linezolid & - & - & - & - & - & - & - & - & $\begin{array}{l}4 \\
(40)\end{array}$ & $\begin{array}{l}4 \\
(100)\end{array}$ & $\begin{array}{l}4 \\
(100)\end{array}$ & $\begin{array}{l}1 \\
(25)\end{array}$ \\
\hline Polymyxin B & $\begin{array}{l}1 \\
(1.04)\end{array}$ & $\begin{array}{l}1 \\
(100)\end{array}$ & $\begin{array}{l}2 \\
(33.3)\end{array}$ & $\begin{array}{l}2 \\
(100)\end{array}$ & - & - & - & - & - & - & - & - \\
\hline
\end{tabular}

$* \mathrm{~T}=$ Tested

$* * \mathrm{~S}=$ Sensitive 
different samples of critically ill patients after $48 \mathrm{hrs}$ of admission to identify hospital acquired infections.

In this study, the infection rate among ICU patients was $28.2 \%$, which though high, was within the reported range $(2.8 \%-34.6 \%) .^{10}$ The high rate of nosocomial infections observed in this study could be due to different clinical profiles of the patients and the absence of a powerful hospital acquired infection control program. Urinary tract infection was the most common infection (54.9\%), followed by respiratory tract $(11 \%)$. In total, predominant organisms isolated were E. coli $(52.7 \%)$, followed by $P$. mirabilis (15.4\%), Ps. aeruginosa (13.2\%), Candida albicans (6.6\%), S. aureus (5.5\%), K. pneumonia $(3.3 \%)$, E. fecalis $(2.2 \%)$. These findings were comparable to the observation of previous studies, where the predominant organism was E.coli. ${ }^{11,12}$ In the ICU of a tertiary care hospital in South India, $K$. pneumoniae and $P S$. aeruginosa were the commonest isolated organisms. ${ }^{10} \mathrm{~A}$ study conducted in 12 ICU's in seven Indian cities showed Enterobacteriaceae (46\%), Pseudomonas (27\%), Acinetobacter spp. (6\%), Candida spp. (8\%), S. aureus $(6 \%)$ as causative agents of nosocomial infections. ${ }^{13}$

In the present study, E. coli was highly sensitive to Polymyxin B, Gatifloxacin, Ceftriaxone, which is contrary to a community based surveillance in $2009^{14}$ and completely resistant to Cephalexin, Cefadroxil, Tobramycin and Prulifloxacin which is consistent with another study of Ibrahim Medical College and Birdem ICU, where the E. coli isolates were highly resistant (>80\%) to Cephalosporins. ${ }^{15} P$. mirabilis and Ps. aeruginosa were sensitive to Gatifloxacin, relatively sensitive to Imipenem, completely resistant to Cefuroxime, Ceftazidime, Cefixime, Cefpodoxime. The Aminoglycosides were totally ineffective against $P$. mirabilis; Pseudomonas demonstrated a high degree of resistance to the third generation Cephalosporins $(>60 \%)$ and the Aminoglycosides, which correlates with a study showing the emergence of antibiotic resistant Pseudomonas by Arora et al. ${ }^{16} \mathrm{~K}$. pneumoniae also showed resistance to most of the antibiotics, but was highly sensitive to Piperacillin-Tazobactum, Sparfloxacin and Levofloxacin. The gram positive cocci $S$. aureus and E. fecalis were highly sensitive to Vancomycin, Linezolid and Amoxicillin-Clavulanic acid, but showed high degree of resistance to Cephalosporins, which supports the claim of Shalini et al. ${ }^{10}$ The high degree of resistance seen to Cephalosporins was probably due to the extensive use of these drugs in the ICU of the hospital.

There were some limitations to this study, because patients who were in the incubation period of nosocomial infections on discharge from the ICU, who manifest it after discharge, were not included in the current study. Contribution of their load to current study prevalence is unknown.

\section{CONCLUSION}

The present study on the bacteriological profiles of the nosocomial infections showed that the rate of such infections is high, even though it was within the reported range. The risk of development of nosocomial infections was directly related to the duration of ICU stay and the duration of the use of the indwelling catheters/tubes. The prolonged use of indwelling devices need careful prophylactic standards of microbiologic monitoring. ${ }^{17}$

Resistance to antibiotics poses a serious and growing problem, because such resistant bacteria are becoming more difficult to treat. The empirical and the indiscriminate use of antibiotics should be avoided in order to curtail the emergence and the spread of drug resistance among nosocomial pathogens.

Reduction of nosocomial infections and antimicrobial resistance is both a challenge and goal of all ICU's around the world. Strict infection control measures like universal precautions and stringent adherence to hand washing practices, formulation of antibiotic policy, surveillance activities, might be required for the same.

Funding: No funding sources

Competing interests: None declared

Ethical approval: Not required

\section{REFERENCES}

1. Shehabi AA, Baadran I. Microbial infection and antibiotic resistance patterns among Jordanian intensive care patients. Eastern Mediterranean Health Journal 1996;2:515-20.

2. Ducel G, Fabry J, Nicolle L, editors. Prevention of hospital acquired infections: A practical guide, 2nd ed Geneva: World Health Organization, 2002.

3. Radji M, Fauziah S, Aribinuko N. Antibiotic sensitivity pattern of bacterial pathogens in the intensive care unit of Fatmawati Hospital, Indonesia. Asian Pacific Journal of Tropical Biomedicine 2011;1:39-42.

4. Tullu MS, Deshmukh CT, Baveja SM. Bacterial profile, antimicrobial susceptibility pattern in catheter related nosocomial infections. J Postgrad Med 1998;44:7-13.

5. Vincent JL, Rello J, Marsall J, Silva E, Anzeuto A, Martin CD, et al. International study of prevalence and outcomes of infection in intensive care units. JAMA 2009;302:2323-9.

6. Ganguly NK, Arora NK, Chandy SJ, Fairoze MN, Gill JP, Gupta U, et al. Rationalizing Antibiotic use to limit antibiotic resistance in India. Indian $\mathrm{J}$ Med Res 2011;134:281-94.

7. Wikipedia, Antibiotic Resistance. Wikipedia Foundation Inc; [Updated 2012 July 29; Cited 2012, July 30].

8. Wattal C, Goel N, Oberoi JK, Raveendran R, Datta S, Prasad KJ. Surveillance of multidrug resistant 
organisms in tertiary care hospital in Delhi, India. J Assoc Physicians India 2010;58 Suppl:32-6.

9. Akash D, Ghildiyal R, Kandian S, Shinkra N. Clinical and Microbiological profile of nosocomial infections in the pediatric intensive care unit. Indian Pediatr 2004;41:1238-46.

10. Shalini S, Kranthi K, Gopalkrishna BK. The microbiological profile of nosocomial infections in the intensive care unit. J Clin Diagn Res 2010;4:3109-12.

11. Zaveri JR, Patel SM, Nayak SN, Desai K, Patel P. A study on bacteriological profile and drug sensitivity and resistance pattern of isolates of the patients admitted in ICUs of a tertiary care hospital in Ahmadabad. NJMR;2:330-4.

12. Patwardhan RB, Dhakephalkar PK, Niphadkar KB, Chopade BA. A study on nosocomial pathogens in ICU with special reference to multiresistant Acinetobactor baumannii harbouring multiple plasmids. Indian J Med Res 2008;128:178-87.

13. Mehta A, Rosenthal BD, Mehta Y, Chakravarthy M, Todi SK, Sen M, et al. Device associated nosocomial infection rates in intensive care units of seven Indian cities (INICC). J Hosp Infect 2007;67:168-74.

14. Holloway K, Mathai E, Sorensen TL, Gray A. Community based surveillance of antimicrobial use and resistance in resource constrained settings: Report on five pilot projects. Geneva: World Health Organization; 2009.

15. Barai L, Fatema K, Ashraful Haq J, Omar Faruq M, Areef Ahsan ASM, Golam Morshed MAH, et al. Bacterial profile and their antimicrobial resistance pattern in an intensive care unit of a tertiary care hospital in Dhaka. Ibrahim Med Coll J 2010;4:66-9.

16. Arora D, Jindal N, Kumar R. Emerging antibiotic resistance in Pseudomonas-A challenge. Int $\mathbf{J}$ Pharmacy Pharmaceutical Sci 2011;3:82-84.

17. Rosenthal VD, Maki DG, Salomao R, Moreno CA, Mehta $\mathrm{Y}$, et al. Device associated nosocomial infections in 55 intensive care units of 8 developing countries. Ann Intern Med 2006;145:582-91.

doi:10.5455/2319-2003.ijbcp20130307

Cite this article as: Pattanayak C, Patanaik SK, Datta PP, Panda P. A study on antibiotic sensitivity pattern of bacterial isolates in the intensive care unit of a tertiary care hospital in Eastern India. Int J Basic Clin Pharmacol 2013;2:153-9. 